\title{
What is Abstinence? Definitions and Examples of Abstinence, to Prevent the Sexual Transmission of the HIV Virus, According to Spanish University Students
}

\author{
Montserrat Planes, Ana B. Gómez and Eugenia Gras \\ Department of Psicology, University of Girona, Spain \\ Mark J. Sullman \\ School of Psychology, Hertfordshire University, United Kingdom \\ Sílvia Font-Mayolas, Mónica Cunill and Maria Aymerich \\ Department of Psicology, University of Girona, Spain
}

\begin{abstract}
Research carried out in several Anglo-Saxon countries shows that many undergraduates identify oral sex and anal sex as examples of abstinent behaviour, while many others consider kissing and masturbation as examples of having sex. The objective of this research was to investigate whether a sample of Spanish students gave similar replies. Seven hundred and fifty undergraduates (92\% aged under $26,67.6 \%$ women) produced examples or definitions of the term 'abstinence'. Spanish students made similar errors to those observed in the Anglo-Saxon samples, in that behaviours that were abstinent from a preventive point of view (masturbating and sex without penetration) were not considered as such, while a number of students reported oral sex as abstinent behaviour. The results suggest that the information on risky and preventive sexual behaviour should cease to use ambiguous or euphemistic expressions and use vocabulary that is clear and comprehensible to everyone.
\end{abstract}

Keywords: sexual abstinence, terminology, AIDS prevention, gender differences.

The use of clear and unambiguous terms is vitally important if preventive campaigns are to be effective in helping interested people to protect their health. Practising abstinence to prevent the sexual transmission of the Human Immunodeficiency Virus (HIV), which causes Acquired Immuno Deficiency Syndrome (AIDS), has been one of the strategies most strongly recommended to young people since the beginning of the pandemic (Barnett \& Parkhurst, 2005; UNAIDS, 2006). However, research carried out with university students from different countries has shown that despite their privileged cultural education, many members of this group mistakenly believe that anal sex and oral sex are examples of abstinent behaviour (Horan, Phillips, \& Hagan, 1998). Conversely, a considerable number of university students

Address for correspondence: Montserrat Planes, Department of Psychology, University of Girona, Emili Grahit, 77, 1707, Girona, Spain.Email: montserrat.planes@udg.edu

Behaviour Change | Volume 26 | Number 3 | 2009 | pp. 1-?? 
think that they are having sex if they touch their partner's breasts or genitals (Pitts \& Rahman, 2001; Randall \& Byers, 2003; Ritchters \& Song, 1999; Sanders \& Reinisch, 1999); behaviours that, from the point of view of preventing sexually transmitted diseases (Centers for Disease Control and Prevention (CDC), 1994), would be considered examples of abstinence. In addition, the term abstinence has religious and moral connotations for many people, meaning that they reject even what would be considered abstinent behaviour, such as kisses and touching. There is also the fear that these are the first steps towards coitus, due to the sexual excitement they can produce (Goodson, Suther, Pruitt, \& Wilson, 2003).

The terms that are used in English (abstinence) and in Spanish (abstinencia) are very similar graphically and phonetically and come from a shared culture. However, it is possible that their meanings vary according to the country and the ethnic groups involved (Bogart, Cecil, Wagstaff, Pinkerton, \& Abramson, 2000).

The current study aimed to find out: (1) whether Spanish students also held inaccurate beliefs about the meaning of abstinence, in terms of AIDS prevention, and (2) whether there were differences according to gender, age and sexual experience.

\section{Methods}

\section{Participants}

The participants were all first and second year students studying at a university in the northeast of Spain. Of the sample of 1123 , obtained through a proportional, multistage sample, 750 agreed to take part, giving a participation rate of $66.78 \%$. More than half of the participants were women $(67.6 \%), 65.5 \%$ were aged between 17 and 20, 26.5\% between 21 and 25 and $8 \%$ were over 25 . The majority of the women $(85.8 \%)$ and men $(82.6 \%)$ had previously had sexual relations.

\section{Materials and procedure}

In 2005, the students in the sample were asked for their voluntary and anonymous collaboration in answering several questionnaires on preventative sexual behaviour. The information was collected during the last 30 minutes of class, having been given authorisation by the teaching staff in charge. The data referring to the meaning of abstinence was collected using the following question: "We would be grateful if you would give us one or more examples or definitions of what abstinence means to you with regards to preventing the sexual transmission of the AIDS virus. You may use the vocabulary you prefer and that you feel most comfortable with'. Below there were three spaces for their responses. The open question format was chosen to explore the qualitative aspects of participants' responses (Horan et al., 1998). This format provided information about which behaviours the participants remembered most easily and therefore which behaviours were more likely to influence his or her behaviour. Furthermore, unlike providing a list of examples, this method of data collection does not induce or confine the participants' responses.

The various examples or definitions were carefully examined for common themes or representations, which led to the responses being placed into 14 different categories (see Table 1). The digressions category consisted of responses that do not actually refer to the meaning of abstinence, but expressed negative opinions about its practice ('it's a pain', 'it's stupid', 'it's boring', etc.). The euphemisms category consisted of responses using colloquial language or slang ('fasting', 'going without', etc.). In the religious or moral connotations category, responses concerning chastity, celibacy, virginity, priests, and so on, 
TABLE 1

Definitions of the Meaning of the Term Abstinence, by Gender \% ( $n$ )

\begin{tabular}{lccc}
\hline Abstinent behaviour & $\begin{array}{c}\text { Male } \\
(n=243) \\
(\mathrm{R}=245)\end{array}$ & $\begin{array}{c}\text { Female } \\
(n=507) \\
(\mathrm{R}=533)\end{array}$ & $\begin{array}{c}\text { Total } \\
(n=750) \\
(\mathrm{R}=778)\end{array}$ \\
\hline Not having sex & $48.1(117)$ & $62.3(316)$ & $57.7(433)^{* *}$ \\
Digressions & $25.5(62)$ & $16.4(83)$ & $19.3(145)^{* *}$ \\
Not having sex with penetration & $2.9(7)$ & $6.7(34)$ & $5.5(41)^{*}$ \\
Euphemisms & $3.7(9)$ & $3.9(20)$ & $3.9(29)$ \\
Not having sexual pleasure & $4.1(10)$ & $3.4(17)$ & $3.6(27)$ \\
Not having sex without penetration & $4.1(10)$ & $3.0(15)$ & $3.3(25)$ \\
Religious and moral connotations & $3.7(9)$ & $3(15)$ & $3.2(24)$ \\
Masturbating & $4.9(12)$ & $1.4(7)$ & $2.5(19) * *$ \\
Not masturbating & $1.6(4)$ & $2.2(11)$ & $2(15)$ \\
Not having physical contact & $0.8(2)$ & $1.6(8)$ & $1.3(10)$ \\
Not having oral sex & $0.8(2)$ & $0.4(2)$ & $0.5(4)$ \\
Sex without penetration & $0.4(1)$ & $0.4(2)$ & $0.4(3)$ \\
Oral sex & $0.0(0)$ & $0.4(2)$ & $0.3(2)$ \\
Don't know & $0.0(0)$ & $0.2(1)$ & $0.1(1)$ \\
No reply a & $11.1(27)$ & $4.5(23)$ & $6.7(50)^{* *}$ \\
\hline Note: $n=$ number of & & & \\
\end{tabular}

Note: $n=$ number of participants; $\mathrm{R}=$ total replies given by the participants. The number of $n$ does not coincide with the number of $\mathrm{R}$, as each participant could give more than one reply. a This category includes the participants who did not give any examples or definitions. $* p<.05 ; * *<.01$

were placed. The remaining categories are sufficiently descriptive and do not require any explanation. In most cases the students used practically the same words as given in Table 1. To investigate any differences according to gender, age or sexual experience, chi-square contingency tests were used.

\section{Results}

In total there were 778 examples or definitions of abstinence, with an average of 1.04 responses per student. Only $11.1 \%$ of the men and $4.5 \%$ of the women did not give any examples or definitions.

The most frequent reply was not having sex, which together with the specification that there should be no penetration (without indicating between which organs or parts of the body) and not having oral sex, was given by almost two thirds of the sample (63.7\%; see Table 1). There were also two main types of mistaken definitions of the term abstinence. One overly strict one, which included the definitions with religious or moral connotations, in addition to not masturbating, not having physical contact, not having sexual pleasure and not practising sex without penetration. The second category could be considered, from a preventive point of view, to be excessively lax, as it included oral sex as an example. Although the negative view of abstinence predominates, as the participants mainly refer to behaviour that should not be done, it should be pointed out that several people mentioned activities that fit in perfectly with the preventive sense of the word, such as masturbation and having sex without penetration.

Behaviour Change 
It should be pointed out that there were a high number of participants, mostly males, whose responses fitted into the digressions category and expressed their lack of conformity with abstinence as a preventative option. Furthermore, only one woman admitted without reservation that she did not know how to define it. There were also quite a few young persons - mostly males — who did not answer this question.

With regard to gender differences, women reported the definition of abstinence as not having sex or not having sex with penetration significantly more often than men did, while a higher percentage of men mentioned masturbation, digressed or did not give any kind of response.

There were age differences among those who digressed. For both genders, young people from 17 to 20 were different to those aged over $25\left(\chi_{1 \text { (men })}^{2}=11.08, p=.001\right)$; $\chi_{1 \text { (women) }}^{2}=9.99, p=.002$ ), with the older ones giving more digressive responses. Among the men, the responses given by those aged between 17 and 20 were significantly different to those given by young people aged between 21 and $25\left(\chi_{1}^{2}=4.54, p=\right.$ .033) and the tendency was again that the older the students, the more they digressed. The same pattern was also observed when comparing women aged between 21 and 25 with those aged over $25\left(\chi_{1}^{2}=7.52, p=.006\right)$. Once again, it was the older women who more frequently digressed.

In the case of masturbation, a similar pattern was found among men: the differences were found between those aged over 25 and those aged between 17 and $20\left(\chi_{1}^{2}=5.25\right.$, $p=.02)$ and those aged between 21 and $25\left(\chi^{2}=4.02, p=.05\right)$. In both cases, the older men were the ones who gave this response in a greater proportion.

The analysis carried out according to whether the participants had had sex or not, differentiating between men and women, showed no significant differences. Both the men and the women who had had sex gave similar definitions to those who had not had sex.

\section{Discussion}

Most of the students defined abstinence by using culturally correct, although not very precise language (i.e., not having sex), except in the case of 45 students (a greater percentage of women) who referred more explicitly to avoiding engaging in sexual relations with penetration and/or oral sex. It should also be pointed out that many young people, especially males, did not respond to this question. There were really very few participants who gave clear examples of abstinent sexual behaviour (19 people mentioned masturbation and three sex without penetration). However, fortunately there were also very few who gave definitions that had the potential to expose the participant to the HIV virus or other sexually transmitted diseases (two women mentioned oral sex). Although oral penetration or oral contact with the genitals does not involve the same degree of risk, in terms of the transmission of the HIV virus or other sexually transmitted diseases, as vaginal or anal penetration (??Remez, 2000), this is still possible, as has been stated in several well-documented cases (Robinson \& Evans, 1999). With regard to the mistaken responses that do not involve the risk of sexually transmitted diseases (e.g., not having sexual pleasure, not having sex without penetration), although for the moment they can be considered to be totally protective, it is not likely that these behaviours can be maintained in the long-term. Therefore, it would be a good idea for young people to know that preventive abstinence is not at odds with sexual pleasure and that it permits any type of sexual activity, except those that involve penetration. 
A surprisingly high proportion of young people (mainly males and those who are older) gave negative digressive responses on the practice of abstinence as a preventive behaviour. This suggests that for these young people abstinence, as they understand it, is not an acceptable preventative option.

If the results obtained here, using open format questions, are compared with those of Horan et al. (1998), collected using a list of 12 possible examples of abstinence, a number of similarities can be seen. Both groups consider, to a greater or lesser degree, that masturbation and oral sex are examples of abstinent behaviour. Furthermore, both groups reported that sexual relations, and more specifically sexual relations with penetration, are not examples of abstinent behaviour. Therefore, the mistaken belief about oral sex being a type of abstinent behaviour was present in both samples. The results found here also supports research by Sanders and Reinisch (1999), who reported that almost two thirds of the North American students they surveyed did not consider oral sex to be having sex. The current findings also support those of Bogart et al. (2000), who found that slightly less than half of the North American students they surveyed mistakenly reported that oral sex was an example of abstinent behaviour. With regards to other countries, Pitts and Rahman (2001) found that two thirds of their sample of UK university students gave this response, while Randall and Byers (2003) found that less than a quarter of their sample of Canadian university students considered oral sex to be having sex. Finally, in a sample of Australian university students Ritchters and Song (1999) found that just under half of their participants held this belief.

In contrast to most of the previously mentioned research no participants in the present study referred directly to anal sex, although there is the possibility that this behaviour was classified by the participants as a generic part of sexual relations or sexual relations with penetration. Taking this same logic further, it is also possible that when reporting sex without penetration as an example of abstinence, a number of participants were also referring to oral sex. However, unlike Horan et al.'s (1998) research, which found the sexually active group had more correct knowledge, the present study found no difference in the definitions of abstinence according to the participants' sexual experience.

In comparison to other research carried out on the same type of population and dealing with the same matter, the participation rate in the present study was greater than the 58\% obtained by Sanders and Reinisch (1999), but was lower than the 97\% reported by Horan et al. (1998). However, many of the students in Horan et al.'s sample were required to participate in the research.

One possible limitation of this study, with reference to the validity of the responses, could be related to the level of replies to the meaning of the term 'preventive abstinence' (an average of 1.10 responses). While this was greater than that obtained in the previous question in the questionnaire (an average of 0.84 responses), it was lower than that observed in the following question (an average of 1.38 responses). However, the number of participants who did not give any response followed the same pattern: 110 students did not reply to the previous question and 55 acted in the same way with regards to the following question in the questionnaire. Therefore, it would seem that the level of responses observed was not affected by a lack of interest in collaborating in the study or boredom. However, with regard to the generalisation of the results, it is possible that the responses of the participants differed significantly from those who did not participate. It seems likely that those people who voluntarily provided information about these controversial, delicate matters were

Behaviour Change 
those who were more aware and held more favourable attitudes towards prevention than those who refused to collaborate.

The results obtained here, together with those of the researchers mentioned above, illustrate that there is not currently an accurate understanding of the terminology used to disseminate preventive messages. Therefore, information about risky sexual behaviour and about possible forms of prevention should cease to use ambiguous or euphemistic expressions and use terms that are clear and understandable to everyone. The terminology used by the CDC (Horan et al., 1998) recommends abstaining from engaging in sexual activities that involve vaginal, anal or oral sexual relations, which is fairly close to what they actually want to be communicated, but still this leaves some aspects open to misinterpretation, as they do not specify the different parts of the body that should not come into contact.

\section{References}

Bogart, L.M., Cecil, H., Wagstaff, D.A., Pinkerton, S.D., \& Abramson, P.R. (2000). Is it 'sex'? College students' interpretations of sexual behavior terminology. The Journal of Sex Research, 37(2), 108-116.

Barnett, T., \& Parkhurst, J. (2005). HIV/AIDS: Sex, abstinence, and behaviour change. The Lancet, 5, 590-593.

Centers for Disease Control \& Prevention (1994). Abstinence, condoms and safer sex: Sorting out the confusion. CDC HIVIAIDS Prevention, 5(1), 7-8.

Goodson, P., Suther, S., Pruitt, B.E., \& Wilson, K. (2003). Defining abstinence: Views of directors, instructors, and participants in abstinence-only-until-marriage programs in Texas. The Journal of School Health, 73(3), 91-96.

Horan, P., Phillips, J., \& Hagan, N. (1998). The meaning of abstinence for college students. Journal of HIV/AIDS Prevention E⿱ Education for Adolescents E Children, 2(2), 51-66.

Pitts, M., \& Rahman, Q. (2001). Which behaviors constitute 'having sex' among university students in the UK? Archives of Sexual Behavior, 30(2), 169-176.

Randall, H.E., \& Byers, E.S. (2003). What is sex? Students' definitions of having sex, sexual partner, and unfaithful sexual behaviour. The Canadian Journal of Human Sexuality, 12(2), 87-96.

??Remez, L. (2006). Oral sex among adolescents: Is it sex or is it abstinence? Family Planning Perspectives, 32 (6), 298-304.

Robinson, E.K., \& Evans, B.G. (1999). Oral sex and HIV transmission. AIDS, 13(6), 737-738.

Ritchters, J., \& Song, A. (1999). Australian university students agree with Clinton's definition of sex. British Medical Journal, 318, 1011-1012.

Sanders, S.A., \& Reinisch, J.M. (1999). Would you say you 'had sex' if ...? Journal of American Medical Association, 281(3), 275-277.

UNAIDS (2006). Q\&AII: Selected issues: prevention, care and funding. Retrieved January 14, 2007, from http://data.unaids.org/pub/GlobalReport/2006/20060530-qa_part2_en.pdf?preview $=$ true. 